\title{
The Proof of the Generalized Piemann's Hypothesis
}

\author{
S. V. Matnyak \\ Khmelnytsky, Ukraine \\ Email: matn111@ukr.net
}

How to cite this paper: Matnyak, S.V. (2018) The Proof of the Generalized Piemann's Hypothesis. Advances in Pure Mathematics, 8, 672-686.

https://doi.org/10.4236/apm.2018.87040

Received: April 16, 2018

Accepted: July 27, 2018

Published: July 30, 2018

Copyright (C) 2018 by author and Scientific Research Publishing Inc. This work is licensed under the Creative Commons Attribution International License (CC BY 4.0).

http://creativecommons.org/licenses/by/4.0/

\begin{abstract}
The article presents the proof of the validity of the generalized Riemann hypothesis on the basis of adjustment and correction of the proof of the Riemanns hypothesis in the work [1], obtained by a finite exponential functional series and finite exponential functional progression.

\section{Keywords}

Riemann's Hypothesis, Natural Series, Function of Möbius, Mertens Function, Finite Exponential Functional Series, Finite Exponential Functional Progression
\end{abstract}

\section{Introduction}

In this paper we give the proof of the generalized Riemann's hypothesison the basis of adjustments and corrections to the proof of the Riemann's hypothesis of the zeta function, which was undertaken in [1], as well as values of specified limits of the cofficient $c$. The paper also provides a refutation of the hypothesis of Mertens.

The formulation of the problem (Generalized Riemann's hypothesis). All non-trivial zeros of Dirichle's function $L(s, \chi)$ have a real part that is equal to $\sigma=\frac{1}{2}$.

1). For this we first will provethe Riemann's hypothesis for the zeta function $\varsigma(n)$.

1.1). The solution. For the confirmation of the Riemann's hypothesis we will give the definitions and prove the following theorem.

Definition 1. The expression

$$
\sum_{k=1}^{[\sqrt{n}]} u^{\frac{1}{k}}(x)=u(x)+u^{\frac{1}{2}}(x)+u^{\frac{1}{3}}(x)+\cdots+u^{\frac{1}{[\sqrt{n}]}}(x)
$$


is called finite exponential functional series with respect to the variable exponent $\frac{1}{k}$, where $k=\{1,2,3, \cdots,[\sqrt{n}]\}$.

Definition 2. The progression of the type

$$
a(x), a(x) \cdot q(x), a(x) \cdot q^{2}(x), \cdots, a(x) \cdot q^{\sqrt{x}}(x)
$$

is called finite exponential functional progression, if their first member $a(n)$ is a function of $x$ or is equal to 1 and the denominat or $q(n)$ is a function of the variable $x^{\frac{1}{\sqrt{x}}}$.

Theorem 1. If the set of natural numbers $N_{n}^{+}=\{1,2, \cdots, k, \cdots, n\}$ is the union of subsets $M_{1}, M_{2}, M_{3}, M_{4}, M_{5}, M_{6}$ and these subsets are disjoint and have appropriately for $m, r, s, t, v, u$ the elements, the number of elements of the set $N_{n}^{+}=M_{1} \cup M_{2} \cup M_{3} \cup M_{4} \cup M_{5} \cup M$ equal to

$$
n=m+r+s+t+v+u \text {. }
$$

Proof. The theorem is proved similarly to the theorem 7.11 ([2], p. 50).

Theorem 2. $M(n)=\sum_{k=1}^{\infty} \mu(k)$ of a series $\frac{1}{\varsigma(s)}=\sum_{n=1}^{\infty} \frac{\mu(n)}{n^{s}}$ equals.

$$
|M(n)|<1.5 \sqrt{n}
$$

Proof. Let the number $N=N(n)$ be the quantity of elements of the set of natural numbers $N_{n}^{+}=\{1,2,3, \cdots, n\}$. The positive integers $N_{n}^{+}$consist: of 1 ; primes, the quantity of which is denoted by $\Pi=\Pi(n)$; of natural numbers, which are divided on $p^{m}$ with quotient from 1 with $m \geq 2$, the number of which is denoted by $K^{\kappa}=K^{\kappa}(n)$; the number of positive integers, which are decomposed into a product of a pair number of primes, we denote by $T^{n}=T^{n}(n)$, and the amount of numbers, which can be converted into product of unpaired number of primes, will be denoted by $T^{\mu}=T^{\mu}(n)$. Then the amount of natural numbers $N$, of the set of natural numbers $N_{n}^{+}$, according to the theorem 1, equals

$$
N_{n}^{+}=1+\Pi+T^{n}+T^{H}+K^{\kappa}+K
$$

The number of the natural series $N_{n}^{+}$approximately can be expressed as a finite exponential function series

$$
f_{1}(n)=\sum_{k=2}^{[n]} n^{\frac{1}{k}}=\sqrt{n}+\sqrt[3]{n}+\sqrt[4]{n}+\cdots+\sqrt[[n]]{n}
$$

We will write the number of natural numbers $K(n)$, approximately as finite exponential function series consisting of the first $(\sqrt{n}-1)$ members of the series (4), and we denote it as $f_{2}(n)$, then

$$
f_{2}(n)=\sum_{k=2}^{[\sqrt{n}]} n^{\frac{1}{k}}=\sqrt{n}+\sqrt[3]{n}+\sqrt[4]{n}+\cdots+[\sqrt[\sqrt{n}]{n}]
$$


In the sum of the series (4) each term of the series is taken, as the amount of natural numbers. For example, $\sqrt[k]{n}=[\sqrt[k]{n}] ;[\sqrt{100}]=\{1 ; 2 ; 3 ; 4 ; 5 ; 6 ; 7 ; 8 ; 9 ; 10\}$; $[\sqrt[3]{100}]=\{1 ; 2 ; 3 ; 4\}$ and so on.

A series $(\sqrt{n}+\sqrt[3]{n}+\sqrt[4]{n}+\cdots+\sqrt[[n]]{n})$ is greater than the series of the natural number of positive integers $f_{1}(n)>N_{n}^{+}$.

Definition 3. The natural numbers that overlap, are called the finite exponential function series of (5), in which they occurmore than once. Let that $N=n$ and $\frac{1}{\sqrt{n}}=\frac{1}{[\sqrt{n}]}$. The function $f_{2}(n)>K(n)$, because in the function except the numbers $K(n)$ are included and the numbers which overlap.

Definition 4. Two infinitely great $f(n)$ and $\varphi(n)$, which are not equal to each ether $f(n) \neq \varphi(n)$ are called equivalent if $\lim _{n \rightarrow \infty} \frac{f(n)}{\varphi(n)}=1$ when $\varphi(n) \neq 0$.

We assume that the set of natural numbers $M_{1}=(1,2,3, \cdots, n-1, n)$ with algebra $A_{1}=\langle N,+, \cdot,-, 0,1\rangle$ is vector space $P_{1}$. In this space we set the standard $\|x\|_{n}=\max _{n}|n|=n$, and the set of numbers $M_{2}=(\sqrt[[n]]{n}, \sqrt[(n-1)]{n}, \cdots, \sqrt{n})$ with algebra $A_{2}=\langle\sqrt[[n]]{n},+, \cdot,-, 0,1\rangle$ will be assumed as vector space $P_{2}$ with standard

$$
\|x\|_{\sqrt{n}}=\max _{\sqrt{n}}|\sqrt{n}|=\sqrt{n} .
$$

Then, the denominator of an exponential function of finite progression, which operates in the space $P_{1}$ will take as $q=n^{\frac{1}{n}}$, and the denominator of an exponential function of finite progression, which operates in the space $P_{2}$, as $q=n^{\frac{1}{\sqrt{n}}}$

The finite exponential functional series (4) is approximable by the sum of the finite exponential functional progression

$$
\varphi_{1}\left(n^{\frac{1}{n}}\right)=1+n^{\frac{1}{n}}+n^{\frac{2}{n}}+n^{\frac{3}{n}}+\cdots+n^{\frac{1}{2}}
$$

Proposition 1. The finite exponential functional series (4) and the sum of the finite exponential functional progression (6) are equivalent.

Proof. To prove the equivalence of the finite exponential functional series $f_{1}(n)$ with the finite functional progression $\varphi_{1}\left(n^{\frac{1}{n}}\right)$ the sum of the functional series is written in the form of

$$
\begin{aligned}
& f_{1}(n)=(\sqrt{n}+\sqrt[3]{n}+\cdots+\sqrt[n]{n}) \\
& <\left(n^{\frac{1}{\sqrt{n}}}(n-\sqrt{n}+1)+(\sqrt{n}+\sqrt[3]{n}+\cdots+\sqrt[\sqrt{n}]{n})\right),
\end{aligned}
$$

Then let us write that 


$$
\begin{aligned}
\lim _{n \rightarrow \infty} \frac{f_{1}(n)}{\varphi_{1}\left(n^{\frac{1}{n}}\right)} & =\lim _{n \rightarrow \infty} \frac{(\sqrt{n}+\sqrt[3]{n}+\ldots+\sqrt[n]{n})}{\left(1+n^{\frac{1}{n}}+n^{\frac{2}{n}}+n^{\frac{3}{n}}+\cdots+n^{\frac{1}{2}}\right)} \\
& =\lim _{n \rightarrow \infty} \frac{\sqrt{n} \cdot\left(1+n^{\frac{1}{3}-\frac{1}{2}}+n^{\frac{1}{4}-\frac{1}{2}}+\cdots+n^{\frac{1}{n}-\frac{1}{2}}\right)}{\sqrt{n} \cdot\left(n^{\frac{-1}{2}}+n^{\frac{1}{n}-\frac{1}{2}}+\cdots+1\right)}=1
\end{aligned}
$$

Therefore, in accordance with the definition 4, the functional series and functional progression will be equivalent.

\section{Proposition 1 is proved.}

Proposition 2. The finite exponential functional series (4) and the sum of the finite exponential functional progression (6) $2 \cdot S_{1}(N)$ are equivalent.

Proof. The sum of the finite exponential functional progression $\varphi_{1}\left(n^{\frac{1}{n}}\right)$ can be calculated by the formula

$$
2 \cdot S_{1}(N)=2 \cdot \frac{\sqrt{n}-1}{n^{\frac{1}{n}}-1}>2 \cdot \frac{\sqrt{n}-1}{\frac{2}{\sqrt{n}}}=(n-\sqrt{n})
$$

and then the limit $\lim _{n \rightarrow \infty} \frac{f_{1}(n)}{\varphi_{1}\left(n^{\frac{1}{n}}\right)}$ will be equal to:

$$
\begin{aligned}
& \lim _{n \rightarrow \infty} \frac{f_{1}(n)}{\varphi_{1}\left(n^{\frac{1}{n}}\right)}=\lim _{n \rightarrow \infty} \frac{n^{\frac{1}{\sqrt{n}}}(n-\sqrt{n}+1)+(\sqrt{n}+\sqrt[3]{n}+\cdots+\sqrt[\sqrt{n}]{n})}{n-\sqrt{n}} \\
& =\lim _{n \rightarrow \infty} \frac{\left(n^{\frac{1}{\sqrt{n}}} n-n^{\frac{1}{\sqrt{n}}} \sqrt{n}+n^{\frac{1}{\sqrt{n}}} 1\right)+(\sqrt{n}+\sqrt[3]{n}+\cdots+\sqrt[\sqrt{n}]{n})}{n-\sqrt{n}} \\
& =\lim _{n \rightarrow \infty} \frac{n+\sqrt[3]{n}+\sqrt[4]{n}+\cdots+\sqrt[\sqrt{n}]{n}+1}{n-\sqrt{n}}=1
\end{aligned}
$$

where $\lim _{n \rightarrow \infty} \sqrt[n]{n}=1$ and $0<\sqrt[n]{n}-1<\frac{2}{\sqrt{n}} \quad([3]$, p. 67)

Therefore, in accordance with the definition 4, the functional series (4) and the finite sum $2 \cdot S_{1}$ of the functional progression $(6)$ at $(\sqrt[n]{n}-1) \approx \frac{2}{\sqrt{n}}$, when $n \rightarrow \infty$, will be equivalent.

\section{Proposition 2 is proved.}

From the expression (4) and (7) one can see that $\varphi_{1}\left(n^{\frac{1}{n}}\right)$ is within limit of function 


$$
f_{1}(n) \approx \varphi_{1}\left(n^{\frac{1}{n}}\right) \geq 2 \cdot S_{1}(n)>n-\sqrt{n}
$$

The sum of the finite exponential functional progression (6) with $q=n^{\frac{1}{n}}$ equals $2 \cdot S_{1}(N) \approx(n-\sqrt{n})$. When $(n \rightarrow \infty), S_{1}(N) \rightarrow \infty$. We will compare the function $2 \cdot S_{1}(n)$ with the function $f(n)=N_{n}^{+}$. We find

$$
k_{1}=\lim _{n \rightarrow \infty} \frac{f(n)}{S_{1}(n)}=\lim _{n \rightarrow \infty} \frac{N}{2 \cdot \frac{\sqrt{n}-1}{\sqrt[n]{n}-1}}<\lim _{n \rightarrow \infty} \frac{N}{2 \cdot \frac{\sqrt{n}-1}{\frac{2}{\sqrt{n}}}}=\lim _{n \rightarrow \infty} \frac{N}{n-\sqrt{n}}=1 .
$$

Therefore, $k_{1}<1$.

Lemma 1. The number of natural numbers that overlap is less than $1,5 \sqrt{n}$.

Proof. To prove this proposition let us denotethrough $K^{n}$ is the numbers that occur more than once in the finite exponential functional series (4) when $n \rightarrow \infty$, and use the exponential functional series $f_{2}(n)$.

The series (5) is taken is this form to be because it includes all numbers that overlap. This follows from the expression $2^{\sqrt{n}}>N, \sqrt{n} \cdot \ln 2>\ln N$. Two is taken because it is thes mallest prime number that can not be decomposed into prime factors.

The finite exponential functional series (5) will be replace by the sum of finite exponential functional progression

$$
\varphi_{2}\left(n^{\frac{1}{\sqrt{n}}}\right)=\left(1+\sum_{k=1}^{\frac{\sqrt{n}}{2}} n^{\frac{k}{\sqrt{n}}}\right)
$$

Proposition 3. The finite exponential functional series (5) and the finite exponential functional progression (8) are equivalent.

Proof. The functional series (4) can be written as

$$
f_{1}(n)=\sum_{k=\sqrt{n}}^{n} n^{\frac{1}{k}}+\left(n^{\frac{1}{2}}+n^{\frac{1}{3}}+\cdots+n^{\frac{1}{\sqrt{n}}}\right),
$$

and the functional progression (6) is as

$$
\varphi_{1}\left(n^{\frac{1}{n}}\right)=\left(1+n^{\frac{1}{n}}+n^{\frac{2}{n}}+\cdots+n^{\frac{n-2 \sqrt{n}}{2 n}}\right)+\left(1+\sum_{k=1}^{\frac{\sqrt{n}}{2}} n^{\frac{k}{\sqrt{n}}}\right)
$$

Discard the first members of the series and progression, we find that

$$
f_{2}(n)=n^{\frac{1}{2}}+n^{\frac{1}{3}}+\cdots+n^{\frac{1}{\sqrt{n}}} \text { or } \varphi_{2}\left(n^{\frac{1}{\sqrt{n}}}\right)=\left(1+\sum_{k=1}^{\frac{\sqrt{n}}{2}} n^{\frac{k}{\sqrt{n}}}\right)
$$

We show that 


$$
f_{3}(n)=\sum_{k=\sqrt{n}}^{n} n^{\frac{1}{k}} \text { and } \varphi_{3}\left(n^{\frac{1}{n}}\right)=\left(1+n^{\frac{1}{n}}+n^{\frac{2}{n}}+n^{\frac{3}{n}}+\cdots+n^{\frac{n-2 \sqrt{n}}{2 n}}\right)
$$

are equivalent:

$$
k_{2}=\lim _{n \rightarrow \infty} \frac{f_{3}(n)}{\varphi_{3}\left(n^{\frac{1}{n}}\right)}=\lim _{n \rightarrow \infty} \frac{\sum_{k=\sqrt{n}}^{n} n^{\frac{1}{k}}}{\left(1+n^{\frac{1}{n}}+n^{\frac{2}{n}}+\cdots+n^{\frac{n-2 \sqrt{n}}{2 n}}\right)}=\lim _{n \rightarrow \infty} \frac{n^{\frac{1}{\sqrt{n}}}(n-\sqrt{n}+1)}{2\left(\frac{n-\sqrt{n}}{2}\right)}=1 .
$$

It follows that the finite exponential functional series $f_{2}(n)$ and the finite exponential functional progression $\varphi_{2}\left(n^{\frac{1}{\sqrt{n}}}\right)$ are equivalent.

\section{Proposition 3 is proved.}

To prove the theorem, we introduce the functions series

$$
f_{4}\left(n^{\frac{1}{k_{1}}}\right)=\sum_{k=2 k_{1}}^{[\sqrt{n}]} n^{\frac{1}{k}}=n^{\frac{1}{4}}+n^{\frac{1}{6}}+\cdots+n^{\frac{1}{\sqrt{n}}}
$$

where $k_{1}=\{2,3, \cdots,[\sqrt{n}] / 2\}$. And functional progression

$$
\varphi_{4}\left(n^{\frac{2}{\sqrt{n}}}\right)=\left(1+n^{\frac{2}{\sqrt{n}}}+n^{\frac{4}{\sqrt{n}}}+\cdots+n^{\frac{\sqrt{n}-8}{4 \sqrt{n}}}+n^{\frac{1}{4}}\right) \text {. }
$$

If we express the series (5), as a series $[\sqrt{n}]+[\sqrt[3]{n}]+[\sqrt[4]{n}]+\cdots+[\sqrt[\sqrt[n]{n}]{n}]$, than the series (8) is taken $[\sqrt{n}]+[\sqrt[4]{n}]+[\sqrt[6]{n}]+\cdots+[\sqrt[2 k]{n}]$ is such form so that each element of the series (8) overlaps the each element of the series (5) with unpaired exponents of the root. And then we can write that

$$
[\sqrt{n}]+[\sqrt[4]{n}]+[\sqrt[6]{n}] \cdots+[\sqrt[k]{n}]>[\sqrt[3]{n}]+[\sqrt[5]{n}]+\cdots+[\sqrt[2 k+1]{n}]
$$

Hence the amount of numbers that cover more numbers that overlap.

Proposition 4. The finite exponential functional series (9) and the finite exponential progression (10) are equivalent.

Proof. To prove the equivalence of the finite exponential functional series with the finite exponential functional progression in the form of the relation

$$
\begin{aligned}
& k_{3}=\lim _{n \rightarrow \infty} \frac{f_{4}(n)}{\varphi_{4}(n)}=\lim _{n \rightarrow \infty} \frac{n^{\frac{1}{4}}+n^{\frac{1}{6}}+\cdots+n^{\frac{1}{\sqrt{n}}}}{\left.1+n^{\frac{2}{\sqrt{n}}}+n^{\frac{4}{\sqrt{n}}}+\cdots+n^{\frac{\sqrt{n}-8}{4 \sqrt{n}}}+n^{\frac{1}{4}}\right)} \\
&=\lim _{n \rightarrow \infty} \frac{n^{\frac{1}{4}}\left(1+n^{\frac{1}{6}-\frac{1}{4}}+\cdots+n^{\frac{1}{\sqrt{n}}-\frac{1}{4}}\right)}{n^{\frac{1}{4}} \cdot\left(n^{-\frac{1}{4}}+n^{\frac{2}{\sqrt{n}}-\frac{1}{4}}+\cdots+1\right)}=1
\end{aligned}
$$

Therefore, in accordance with the definition 4, the functional series (9) and a 
functional progression (10) are equivalent.

Proposition 4 is proved.

Proposition 5. The finite exponential functional series (9) and the finite sum $4 \cdot S_{4}(n)$ of the exponential function progression (10) are equivalent when $\sqrt[n]{n}-1 \approx \frac{2}{\sqrt{n}}([3]$, p. 67$)$

Proof. The sum of functional series $f_{4}(n)$ is more than $\left(\frac{\sqrt{n}}{2}-1\right)$, and the sum of functional progression $\varphi_{4}\left(n^{\frac{2}{\sqrt{n}}}\right)$ is considered, as the sum of the functional progression with $q=n^{\frac{2}{\sqrt{n}}}$. Then we find that $4 \cdot S_{4}(n)=4 \cdot \frac{\sqrt[4]{n}-1}{\sqrt[\sqrt[n]{n}]{n^{2}}-1}$. In order to calculate the functional $\left(n^{\frac{2}{\sqrt{n}}}-1\right)$, let us set $t^{2}=n$ or $t=\sqrt{n}$, and then we obtain

$$
\left(n^{\frac{2}{\sqrt{n}}}-1\right)=\left(t^{\frac{4}{t}}-1\right)=\left(t^{\frac{2}{t}}-1\right) \cdot\left(t^{\frac{2}{t}}+1\right)=\left(t^{\frac{1}{t}}-1\right) \cdot\left(t^{\frac{1}{t}}+1\right) \cdot\left(t^{\frac{2}{t}}+1\right) .
$$

Since $\lim _{t \rightarrow \infty} \sqrt[t]{t}=1$ then $\left(n^{\frac{2}{\sqrt{n}}}-1\right)<\frac{8}{\sqrt[4]{n}}$. And then we will have

$$
4 \cdot S_{4}(n) \approx 4 \frac{\sqrt[4]{n}-1}{\frac{8}{\sqrt[4]{n}}}=\frac{\sqrt{n}-\sqrt[4]{n}}{2},
$$

Using the definition 4 we will have

$$
k_{3}=\lim _{n \rightarrow \infty} \frac{f_{4}(n)}{\varphi_{4}\left(n^{\frac{2}{\sqrt{n}}}\right)}=\lim _{n \rightarrow \infty} \frac{\frac{\sqrt{n}}{2}-1}{\frac{\sqrt{n}-\sqrt[4]{n}}{2}}=1 .
$$

Therefore, a function of series (9) and the sum $4 \cdot S_{4}(n)$ of functional progression (10) are equivalent.

Proposition 5 is proved.

From the expressions (10) and (12) it is clear that $\varphi_{4}(n)$ is within

$$
f_{4}\left(n^{\frac{2}{\sqrt{n}}}\right) \approx \varphi_{4}(n) \geq 4 \cdot S_{4}(n)>\frac{\sqrt{n}-\sqrt[4]{n}}{2} .
$$

Then we compare function $\sqrt{n}$ with the function $f_{4}(n)$ when $n \rightarrow \infty$ and we obtain

$$
k_{4}=\lim _{n \rightarrow \infty} \frac{\sqrt{n}}{4 \cdot \frac{\sqrt[4]{n}-1}{\sqrt[\sqrt{n}]{n}-1}}<\lim _{n \rightarrow \infty} \frac{\sqrt{n}}{4 \cdot \frac{\sqrt[4]{n}-1}{\frac{8}{\sqrt[4]{n}}}}=\lim _{n \rightarrow \infty} \frac{8 \cdot \sqrt{n}}{4 \cdot(\sqrt{n}-\sqrt[4]{n})}=2 .
$$

Hence, we have that $k_{4}<2$, or $\sqrt{n}-2 \cdot f_{4}\left(n^{\frac{2}{\sqrt{n}}}\right)<0$. 
Therefore, $\frac{\sqrt{n}}{2}<f_{4}(n)$. Let us take into account the value of the finite exponential functional series $f_{4}(n)$, and write that

$$
\frac{\sqrt{n}}{2}<K^{n}(n)<\sqrt{n}+\frac{\sqrt{n}}{2}=\frac{3}{2} \sqrt{n} .
$$

\section{Lemma 1 is proved.}

Then we can write that

$$
N(n)-c \cdot f_{1}(n)<0,
$$

Using the inequality $f_{1}(n)>2 \cdot K(n)$ we will write that

$$
f_{1}(n) \approx K(n)+(n-\sqrt{n}+1)+K^{n}(n)
$$

If we substitute value of the function $f_{2}(n)$ (14) into (13), we obtain

$$
N(n)-c \cdot\left(K(n)+(n-\sqrt{n}+1)+K^{n}(n)\right)<0 .
$$

Using Lemma 1, we obtain

$$
N(n)-c \cdot\left(K(n)+(n-\sqrt{n}+1)+\frac{3}{2} \sqrt{n}\right)<0 .
$$

Hence; we find that

$$
N(n)-c \cdot(K(n)+(n-\sqrt{n}+1))<c \cdot \frac{3}{2} \sqrt{n} .
$$

The value $N$ from (3) is substituted instead $N$, we obtain

$$
1+\Pi+T^{H}+T^{n}+K^{\kappa}+K(n)<c \cdot(1.5 \sqrt{n}+K(n)+(n-\sqrt{n}+1)),
$$

or

$$
1+\Pi+T^{\mu}+T^{n}+K^{\kappa}<c \cdot\left(1.5 \sqrt{n}+\left(1+\Pi+T^{H}+T^{n}+K^{\kappa}-\sqrt{n}+1\right)\right) .
$$

Then we can write that appropriately of the properties of the function of Mobius- $\mu(n)=1$, when $n=1 ; \mu(n)=(-1)^{k}$, where $k$ is the amount of prime factors of the numbers $n=p_{1} \cdot p_{2} \cdots p_{k}$ and $\mu(n)=0$ when $n$ is multiple $p^{m}$ for $m \geq 2$,

$$
1+T^{n}-\left(\Pi+T^{H}\right)<c \cdot\left(\left(1+T^{n}\right)-\left(\Pi+T^{H}\right)-\sqrt{n}+1+1.5 \sqrt{n}\right) .
$$

We write that

$$
M(n)=1+T^{n}-\left(\Pi+T^{\mu}\right)
$$

Then the expression (16) takes the form

$$
M(n)<c \cdot((M(n)-\sqrt{n}+1)+1.5 \sqrt{n}) .
$$

Therefore

$$
|M(n)|<\frac{c}{c-1} \cdot(0.5 \sqrt{n}+1) .
$$




\section{The theorem is proved.}

For the Mertens function we can find a more precise estimate.

Lemma 2. The accurate assessment. $M(n)=\sum_{k=1}^{\infty} \mu(k)$ in a series $\frac{1}{\varsigma(s)}=\sum_{n=1}^{\infty} \frac{\mu(n)}{n^{s}}$ will be equal

$$
M(n)<\frac{c}{c-1}(1.25 \cdot \sqrt{n}+\sqrt[3]{n}-1.25 \cdot \sqrt[4]{n}-\sqrt{n}+1) .
$$

Proof. In order to finda more accurate estimate than $M(n)<\frac{c}{c-1}(0.5 \sqrt{n}+1)$, let us find the sum of the finite exponential functional series (5) $f_{2}(n)=\sum_{k=2}^{[\sqrt{n}]} n^{\frac{1}{k}}=(\sqrt{n}+\sqrt[3]{n}+\sqrt[4]{n}+\cdots+[\sqrt[[\sqrt{n}]]{n})$. For that we use the functional progression (6) $\varphi_{2}\left(n^{\frac{1}{\sqrt{n}}}\right)=\left(1+\sum_{k=1}^{\frac{\sqrt{n}}{2}} n^{\frac{k}{\sqrt{n}}}\right)$, then we obtain that

$$
f_{2}(n)=\sqrt{n}+\sqrt[3]{n}+\sqrt[4]{n}+\cdots+\sqrt[\sqrt{n}]{n}=2.5 \sqrt{n}+2 \sqrt[3]{n}-2.5 \cdot \sqrt[4]{n} .
$$

We find from the expression (10) that the quantity of numbers that overlap is less than $\frac{f_{2}(n)}{2}$ because $\frac{f_{2}(n)}{2}>[\sqrt[3]{n}]+[\sqrt[5]{n}]+\cdots+[\sqrt[2 k+1]{n}]$. Using this method, we define what $M(n)<\frac{c}{c-1}(0.5 \cdot \sqrt{n}+1)$ and the expression $\frac{f_{2}(n)}{2}>[\sqrt[3]{n}]+[\sqrt[5]{n}]+\cdots+[\sqrt[2 k+1]{n}]$ we obtain that

$$
M(n)<\frac{c}{c-1}(1.25 \cdot \sqrt{n}+\sqrt[3]{n}-1.25 \cdot \sqrt[4]{n}-\sqrt{n}+1) \quad .
$$

Hence, we find that the upper limit of the value functions $\lim _{n \rightarrow \infty} \frac{M(n)}{\sqrt{n}}$ will be the value

$$
\lim _{n \rightarrow \infty} \sup \frac{M(n)}{\sqrt{n}}<\frac{c}{c-1} \cdot 0.25
$$

and the lower limit is

$$
\liminf _{n \rightarrow \infty} \frac{M(n)}{\sqrt{n}}>-\frac{c}{c-1} 0.25 .
$$

Therefore, the evaluation $M(n)<\frac{c}{c-1}(0.25 \sqrt{n}+\sqrt[3]{n}-1.25 \sqrt[4]{n}+1)$ is a more accurate estimate than $M(n)<\frac{c}{c-1}(0.5 \cdot \sqrt{n}+1)$ when $n \rightarrow \infty$.

\section{Lemma 2 is proved.}

The theorem 2 proves that the upper limit value of the function $\lim _{n \rightarrow \infty} \frac{M(n)}{\sqrt{n}}$ equals 


$$
\limsup _{n \rightarrow \infty} \frac{M(n)}{\sqrt{n}}<0.5 \cdot \frac{c}{c-1}
$$

and the lower limit is

$$
\liminf _{n \rightarrow \infty} \frac{M(n)}{\sqrt{n}}>-0.5 \cdot \frac{c}{c-1}
$$

Proposition 6. $\frac{c}{c-1} \cdot(0.5 \sqrt{n}+1) \ll n^{\frac{1}{2}+\varepsilon}$ when $n \rightarrow \infty$.

Proof. According to the theorem $54\left([4]\right.$, p. 114) we have that $M(n)=O\left(n^{\frac{1}{2}+\varepsilon}\right)$.

The value $M(n)=O\left(\frac{c}{c-1} \cdot(0.5 \sqrt{n}+1)\right)$ is compared with $M(n)=O\left(n^{\frac{1}{2}+\varepsilon}\right)$, we will write that $\left(\frac{c}{c-1}(0.5 \sqrt{n}+1)\right)=n^{\frac{1}{2}+\varepsilon_{0}}$ when $n \rightarrow \infty$. Hence we find that $\varepsilon_{0}=\frac{\ln \left(\frac{c}{c-1}(0.5 \sqrt{n}+1)\right)}{\ln n}$ when $n \rightarrow \infty, \varepsilon_{0} \rightarrow 0$. Therefore, we can assume that $\varepsilon>\varepsilon_{0}$, where $\varepsilon$ is a random small number. And here we find that $\frac{c}{c-1} \cdot(0.5 \sqrt{n}+1)<<\left(n^{\frac{1}{2}+\varepsilon}\right)$ when $n \rightarrow \infty$.

\section{Proposition 6 is proved.}

1.2). A determination the values of coefficient $c$.

1.2.1). Then we can write that according to the properties of Mobius function$\mu(n)=1$, then $n=1 ; \mu(n)=(-1)^{k}$, where $k$ the number of prime factors of the number $n=p_{1} \cdot p_{2} \cdots p_{k}$ and $\mu(n)=0$, when $n$ is the multiple of $p^{m}$ for $m \geq 2$ that

$$
\left(\left(1+T^{n}\right)-\left(\Pi+T^{\mu}\right)\right)-c \cdot\left(1+T^{n}-\left(\Pi+T^{\mu}\right)\right)<(0.5 \cdot \sqrt{n}+1),
$$

Then

$$
M(n)<\frac{c}{1-c} \cdot(0.5 \cdot \sqrt{n}+1) ;|M(n)|<\frac{c}{c-1} \cdot(0.5 \cdot \sqrt{n}+1)
$$

From the expression $K(n)+(n-\sqrt{n}+1)+\frac{3}{2} \cdot \sqrt{n}>0$, using the properties of Möbius function, it can be written that

$$
M(n)<1.5 \cdot \sqrt{n}-1 .
$$

And from the expression $N-\sqrt{n}>0$ we find that

$$
M(n)>\sqrt{n} .
$$

This coincides with the results [5]. Then we can find the extent to which the coefficient $c$ is located. From the double inequality $\sqrt{n}<\frac{c}{c-1} \cdot(0.5 \cdot \sqrt{n}+1)<1.5 \cdot \sqrt{n}$, we find that $2<\frac{c}{c-1}<3$. And here we find that $1.5<c<2$. 
1.2.2). Using a more precise value $M(n)$, we find that

$$
\sqrt{n}<\frac{c}{c-1}(0.25 \sqrt{n}+\sqrt[3]{n}-1.25 \sqrt[4]{n}+1)<1.25 \cdot \sqrt{n} .
$$

And here we find that $4<\frac{c}{c-1}<5$ and from the double inequality we find that the coefficient $c$ will be in the range $1.25<c<1.33$.

1.3). Theorem 3. The series $\frac{1}{\varsigma(s)}=\sum_{n=1}^{\infty} \frac{\mu(n)}{n^{s}}$ converges if $\sigma=\frac{1}{2}+\varepsilon>\frac{1}{2}$ and $|M(n)|<\frac{c}{c-1}(0.5 \sqrt{n}+1)$ where $\varepsilon$ is a random small number.

Corollary of Theorem 3 (the Riemann's hypothesis). All non-trivial zeros of the zeta-function have a real partequal to $\sigma=\frac{1}{2}$.

Proof. A necessary and sufficient condition for the validity of the Riemann's hypothesis is the convergence of the series $\frac{1}{\varsigma(s)}=\sum_{n=1}^{\infty} \frac{\mu(n)}{n^{s}}$ when $\sigma>\frac{1}{2}$ ([4], p. 114). We find the convergence of the series, when

$$
\begin{gathered}
M(n)=O\left(\frac{c}{c-1}(0.5 \sqrt{n}+1)\right) \text { and } \sigma=\frac{1}{2} \\
\frac{1}{\xi\left(\frac{1}{2}\right)}=\sum_{n=1}^{\infty} \frac{M(n)}{n^{\frac{1}{2}}}=\sum_{n=1}^{\infty} \frac{M(n)-M(n-1)}{n^{\frac{1}{2}}}=\sum_{n=1}^{\infty} M(n) \cdot\left(\frac{1}{n^{\frac{1}{2}}}-\frac{1}{(n+1)^{\frac{1}{2}}}\right) \\
=\sum_{n=1}^{\infty} M(n)\left(\frac{(n+1)^{\frac{1}{2}}-n^{\frac{1}{2}}}{n^{\frac{1}{2}} \cdot(n+1)^{\frac{1}{2}}}\right) \leq \sum_{n=1}^{\infty} \frac{M(n)}{2 n \sqrt{n}} \\
=\sum_{n=1}^{\infty} \frac{M(n)}{2 n \sqrt{n}}=\sum_{n=1}^{\infty} \frac{c}{c-1}(0,5 \sqrt{n}+1) \\
2 n \sqrt{n}
\end{gathered}
$$

the series diverges.

$$
\begin{aligned}
& \text { And when } \sigma=\frac{1}{2}+\varepsilon>\frac{1}{2} \text { we have } \\
& \qquad \begin{aligned}
\frac{1}{\varsigma\left(\frac{1}{2}+\varepsilon\right)} & =\sum_{n=1}^{\infty} \frac{M(n)}{n^{\frac{1}{2}+\varepsilon}} \leq \frac{0.5 \cdot \frac{c}{c-1}}{2} \sum_{n=1}^{\infty} \frac{1}{n^{1+\varepsilon}} \leq \frac{0.5 \cdot \frac{c}{c-1}}{2} \int_{1}^{\infty} \frac{1}{n^{1+\varepsilon}} \mathrm{d} n \\
& =\frac{0.5 \cdot \frac{c}{c-1}}{2 \varepsilon}=0.25 \cdot \frac{c}{\varepsilon \cdot(c-1)}
\end{aligned}
\end{aligned}
$$

the series converges, where $\varepsilon$ is an arbitrary small number.

Therefore, the series $\frac{1}{\varsigma(s)}=\sum_{n=1}^{\infty} \frac{\mu(n)}{n^{s}}$ converges uniformly for $\sigma=\frac{1}{2}+\varepsilon>\frac{1}{2}$, and since it is a function $\frac{1}{\varsigma(s)}$ if $\sigma>1$, for the theorem of analytic continuation, it is also at its $\frac{1}{2}<\sigma \leq 1$. Therefore, the Riemann's hypothesis is 
true.

The theorem is proved.

\section{Theorem 4. All Non-Trivial Zeros of Dirichle's Function} $L(s, \chi)$ Have a Real Part That Is Equal to $\sigma=\frac{1}{2}$

Proof. Let's consider the Dirichle's series

$$
L(s, \chi)=\sum_{n=1}^{\infty} \frac{\chi(n)}{n^{s}}, s=\sigma+i t
$$

where $\chi$ is the character of modulus $m$.

There is $\varphi(m)$ of such series where $\varphi$ is the Euler's function. Since $|\chi(n)| \leq 1$, the series (18) converges when $\sigma>1$, as can be seen from a comparison of this series with the series $\sum \frac{1}{n^{s}}$. We denote it by the sum through series $L(1, \chi)$. For various characters $\chi$, we obtain different functions $L(s, \chi)$.They are called $L$ is the Dirichle's functions. In studying the properties of these functions it is convenient to distinguish the cases where $\chi$ is the main character $\chi_{1}$ and when $\chi \neq \chi_{1}$.

2.1) If $\chi \neq \chi_{1}$ than the series (18) converges in the half-plane $\sigma>0$. Let us show from the beginning, that the partial sums $\sum_{n<x} \chi(n)$ are limited. We divide the integer number from 1 to $[x]$ into classes of deductions by $\bmod m$ and write $[x]=m \cdot q+r, 0 \leq r \leq m-1$. Then

$$
\sum_{n<x} \chi(n)=\sum_{n=1}^{[x]} \chi(n)=\left(\sum_{1}^{m}+\sum_{m+1}^{2 m}+\ldots+\sum_{m(n-1)+1}^{m q}\right) \cdot \chi(n)+\sum_{m q+1}^{m q+r} \chi(n) .
$$

Because of the orthogonality relations

$$
\sum_{n(\bmod m)} \chi(n)= \begin{cases}\varphi(m), & \text { than } \chi=\chi_{1} \\ 0, & \text { than } \chi \neq \chi_{1}\end{cases}
$$

we have

$$
\sum_{n<x} \chi(n)=\sum_{m q+1}^{m q+r} \chi(n)
$$

hence

$$
\left|\sum_{n<x} \chi(n)\right| \leq \sum_{m q+1}^{m q+r}|\chi(n)| \leq r<m
$$

Since $n^{-\sigma}$ at $\sigma>0$ decreases monotonically and tends to zero when $n \rightarrow \infty$, then the series $\sum \chi(n) / n^{s}$ converges for real $s=\sigma>0$, and, consequently, for all $s$ in the half-plane $\sigma>0$ when $\chi \neq \chi$. If, however, $\sigma<0$, then this the series obviously diverge. It's abscissa converges $\sigma_{0}=0$ and the abscissa of absolute convergence $\bar{\sigma}=1$. By the theorem 4 , The Dirichle's series $\sum_{n=1}^{\infty} a_{n} \cdot n^{-s}$ in the half-plane of the convergence is a regular analytic 
function from $s$, the successive derivatives of which are obtained by the term differentiation of this the series ([6], p. 153), the function $L(s, \chi), \chi \neq \chi$ is a regular analytic function from $s$ when $\sigma>0$.

2.2) If $\chi=\chi_{1}$ we use

$$
L(s, \chi)=\sum_{n=1}^{\infty} \frac{\mu(n) \cdot \chi(n)}{n^{s}}, \operatorname{Re} s \geq \frac{1}{2}
$$

From the theorem 3 it follows that $L(s, \chi) \neq 0$ when $\sigma \geq \frac{1}{2}$. If $\chi_{1}$ is the main character by $\bmod m$, then

$$
\chi_{1}(a)=\left\{\begin{array}{l}
1, \text { then }(a, m)=1 \\
0, \text { then }(a, m)>1
\end{array} .\right.
$$

Using the condition $\left|\chi(n) \cdot n^{-s}\right| \leq n^{-s}$ the function (19) can be written as $L(s, \chi)=\sum_{n=1}^{\infty} \frac{\mu(n) \chi(n)}{n^{s}}=\sum_{n=1}^{\infty} \frac{\mu(n)}{n^{s}}$, when $\chi(n)=1$ and $\sigma \geq \frac{1}{2}$.

Using the results of the theorem 3, it can be argued that the generalized Riemann's hypothesis is true, and accordingly to it: "All non-trivial zeros of the Dirichle's functions have a real part equal to $\sigma=\frac{1}{2}$ ”.

The theorem 4 is proved.

\section{Appendix. Disproof of the Mertens Hypothesis}

The refutation of the Mertens hypothesis can be found on the basis of the proof of the Riemann hypothesis given in this paper. Take the series

$$
f_{1}=(\sqrt{n}+\sqrt[3]{n}+\cdots+\sqrt[n]{n})<\left(n^{\frac{1}{\sqrt{n}}} \cdot(n-\sqrt{n}+1)+(\sqrt{n}+\sqrt[3]{n}+\cdots+\sqrt[\sqrt[n]{n}]{n})\right)
$$

and it can be written in the form

$$
\begin{aligned}
& \left(n^{\frac{1}{\sqrt{n}}} \cdot(n-\sqrt{n}+1)+(\sqrt{n}+\sqrt[3]{n}+\cdots+\sqrt[\sqrt{n}]{n})\right) \\
& <n+2.5 \cdot \sqrt[2]{n}+2 \cdot \sqrt[3]{n}-2.5 \cdot \sqrt[4]{n}-\sqrt{n}+1 \\
& =n+1.5 \cdot \sqrt{n}+2 \cdot \sqrt[3]{n}-2.5 \cdot \sqrt[4]{n}+1,
\end{aligned}
$$

where

$$
f_{2}(n)=\sqrt{n}+\sqrt[3]{n}+\cdots+\sqrt[\sqrt{n}]{n}<2.5 \sqrt{n}+2 \cdot \sqrt[3]{n}-2.5 \cdot \sqrt[4]{n}
$$

Then we can write

$$
N+2.5 \cdot \sqrt{n}+2 \cdot \sqrt[3]{n}-2.5 \cdot \sqrt[4]{n}-\sqrt{n}+1>0
$$

or

$$
N>-1.5 \cdot \sqrt{n}-2 \cdot \sqrt[3]{n}+2.5 \cdot \sqrt[4]{n}-1
$$

The value $N$ from the expression 3 is substituted instead of $N$, we obtain

$$
N=1+\Pi+T^{n}+T^{H}+K^{\kappa}+K(n)
$$


From the expression (20) we obtain

$$
1+\Pi+T^{n}+T^{H}+K^{\kappa}+K(n)>-1.5 \cdot \sqrt{n}-2 \cdot \sqrt[3]{n}+2.5 \cdot \sqrt[4]{n}-1 .
$$

The properties of Mobius function ([5], p. 3) will be applied to the expression (21) and we obtain that

$$
M(n)>-1.5 \cdot \sqrt{n}-2 \cdot \sqrt[3]{n}+2.5 \cdot \sqrt[4]{n}-1
$$

or

$$
M(n)<1.5 \cdot \sqrt{n}+2 \cdot \sqrt[3]{n}-2.5 \cdot \sqrt[4]{n}+1
$$

It will be the smallest value of the function of Mertens $M(n)$ and the biggest value for the function of Mertens $M(n)$. From the expression 6

$$
\phi_{1}\left(n^{\frac{1}{n}}\right)=1+n^{\frac{1}{n}}+\cdots+n^{\frac{1}{2}}
$$

we find that

$$
N-\sqrt{n}>0
$$

When $(\sqrt[n]{n}-1) \approx \frac{2}{\sqrt{n}}$ and $n \rightarrow \infty \quad([3], \mathrm{p} .67)$.

We write the expression 22 in the form

$$
1+\Pi+T^{n}+T^{H}+K^{\kappa}+K(n)>\sqrt{n} .
$$

Let us apply the properties of Mobius function to the expression (23) and we obtain

$$
M(n)>\sqrt{n}
$$

Then we can state that the function of Mertens $M(n)$ is within

$$
\sqrt{n}<M(n)<1.5 \cdot \sqrt{n}+2 \cdot \sqrt[3]{n}-2.5 \cdot \sqrt[4]{n}+1
$$

And it rejects the hypothesis of Mertens.

\section{Conclusion}

In the article, based on the finite exponential functional series and the finite exponential functional progressions, we prove the generalized Riemann's hypothesis, as well as the Riemann hypothesis. It is shown that in the Riemann's hypothesis $\sigma=\frac{1}{2}$. In the annex to the article, the Mertens hypothesis is refuted. In the refuted Mertens hypothesis it is shown that the Mertens function is within $\sqrt{n}<M(n)<1.5 \cdot \sqrt{n}+2 \cdot \sqrt[3]{n}-2.5 \cdot \sqrt[4]{n}+1$.

\section{References}

[1] Matnyak, S.V. (2014) Proof of the Riemann's Hypothesis. arXiv:1404.5872 [math.GM]

[2] Lyapin, Y.S. and Yevseyev, A.Y. (1974) Algebra and Theory of Numbers. Numbers. Educational Book for Students of the Faculties of Physics and Mathematics of Teaching Colleges. M.: "Prosveshcheniye", 383 p. 
[3] Fihtengolc, G.M. (1969) Differential and Integral Calculus Course. V. 1.M.: "Nauka”, 607 p.

[4] Titchmarsh, Y.K. (1947) Riemann Zeta Function. M.: IL, 154 p.

[5] Odlyzko, A.M. and te Riele, H. (1985) Disproof of the Mertens Conjeture. Journal fur die reine und angewandte Mathematik, 357, 138-160.

[6] Chandrasekharan, K. (1974) Introduction Toanalytic Number Theory. M.: Mir, 187 p. 